\title{
Gender Incongruence of Childhood Diagnosis and Its Impact on Brazilian Healthcare Access
}

\author{
Maria Inês Rodrigues Lobato ${ }^{1,2} \cdot$ Alexandre Saadeh $^{3} \cdot$ Desiree Monteiro Cordeiro $^{3}$ • \\ Daniel Augusto Mori Gagliotti ${ }^{3}$ Ângelo Costa Brandelli ${ }^{2,4}$. Anna Martha V. Fontanari ${ }^{1,2}$. \\ César Bridi Filho $^{2}$. Cláudia Corrêa Garcia de Garcia ${ }^{1,2}$ • Dhiordan Cardoso da Silva ${ }^{1,2}$. \\ Jaqueline Salvador ${ }^{1,2} \cdot$ Karine Schwarz $^{1,2}$ (D) Maiko Abel Schneider ${ }^{1,2}$. \\ Márcia Ivani Brambila ${ }^{1,2}$. Aline Giardin ${ }^{1,2}$. Lisia Maya Monteiro ${ }^{1,2}$. \\ Bianca Machado Borba Soll ${ }^{1,2} \cdot$ André Gonzales Real $^{2}$
}

Received: 1 September 2016/ Accepted: 8 September 2016/ Published online: 22 September 2016

(C) Springer Science+Business Media New York 2016

We have read the article by Winter, De Cuypere, Green, Kane, and Knudson (2016) regarding the WPATH statement on the Gender Incongruence of Childhood Diagnosis. Here, we want to express our concern about the opinion of about half of the participating European and North American WPATH members. We feel that the situation of people in other parts of the world has hardly been taken into account.

Only in 1997, Brazilians had the first official regulation by the Federal Medicine Council for the adult population with gender dysphoria (or gender incongruence) and only in 2008 did the Brazilian public healthcare system begin to cover trans-related procedures such as hormone therapy and surgeries. Our clinic started in 1998, and most adults with gender dysphoria/gender incongruence who came to our services were neglected and sometimes abandoned by their families since childhood. They were emotionally and physically abused and had poor or no social support. When they finally arrived at our transgender services, they carried the suffering and health disabilities related to these experiences with them.

Considering these facts, we recognize how much we still need to do for this vulnerable population. This is especially true when we consider the children. There are two services in Brazil

Karine Schwarz

karinesfono@hotmail.com

1 Graduate Program in Medical Sciences: Psychiatry, Universidade Federal do Rio Grande do Sul, Ramiro Barcelos, 2400, Porto Alegre, RS 90035-003, Brazil

2 Gender Identity Disorder Program, Hospital de Clinicas de Porto Alegre, Universidade Federal do Rio Grande do Sul, Porto Alegre, RS, Brazil

3 Hospital de Clínicas da Faculdade de Medicina da Universidade de São Paulo, São Paulo, Brazil

4 Pontifícia Universidade Católica, Porto Alegre, RS, Brazil
(PROTIG-Porto Alegre and AMTIGOS-São Paulo) organized to assist children and adolescents with gender dysphoria/gender incongruence. Both are situated at university hospitals, with multidisciplinary teams. Gender dysphoric children urgently need services to prevent and to reduce the risks associated with the horrifying experiences that impacted so many adults who never had any support from mental health providers. However, a diagnosis of gender dysphoria/gender incongruence in children and adolescents is a formal requirement for their access to the Brazilian public healthcare system that is based on ICD parameters. Therefore, what can be expected with the removal of the Gender Incongruence of Childhood Diagnosis in children? Brazilian children will again be increasingly exposed to neglect or abuse, possibly by their own families, whereas parents who want to help their children will be without any support from health providers. Furthermore, mental health and medical professionals will no longer be appropriately trained. Finally, using the diagnosis will no longer be helpful in schools, who would otherwise be open to special arrangements.

Counseling these children and their families is of utmost importance for the preservation of their self-worth and selfesteem and to overcome experiences of teasing and bullying. For the protection and support of transgender children in our context, that is still marked by severe prejudice and discrimination toward gender and sexuality diversity. It is crucial that the GIC condition remain in the ICD.

\section{Reference}

Winter, S., De Cuypere, G., Green, J., Kane, R., \& Knudson, G. (2016). The proposed ICD-11 gender incongruence of childhood diagnosis: A World Professional Association for Transgender Health Membership Survey. Archives of Sexual Behavior, 45, 1605-1614. 